\title{
A KINEMATICAL TREATMENT OF SOME THEOREMS ON NORMAL RECTILINEAR CONGRUENCES*
}

BY

\author{
CHARLES H. ROWE
}

1. Introduction. When we are discussing the motion of a rigid body in space, we often have occasion to consider the ruled surfaces which are described in space and in the body by the same moving straight line, or, what amounts to the same thing, the surface which a moving straight line actually describes during its motion, and the surface which it appears to describe to an observer who is moving at the same time in an assigned manner. Usually the motions with which we are concerned are ordinary or one-parameter motions, but an analogous idea presents itself at once when we consider instead two-parameter motions. Thus, with each of the $\infty^{2}$ positions of a straight line $l$ which has a two-parameter motion in space, we may associate a position of a rectangular trihedral $T$, and we may imagine an observer (whom also we shall call $T$ ) who shares the two-parameter motion of this trihedral and uses it as his system of reference, estimating each position of the straight line $l$ by referring it to the corresponding trihedral $T$. We can then consider two rectilinear congruences: that which the line $l$ actually describes in space during its two-parameter motion, and that which it appears to describe to the observer $T$. $\dagger$ The second congruence is of course equivalent to that which is described by a line $l^{\prime}$ whose position relative to a trihedral fixed in space is the same as the position of $l$ relative to the corresponding trihedral $T$.

Instead of a straight line which describes a congruence, we may, more generally, consider a geometrical element $e$ of any nature, and if, as before, we associate a trihedral $T$ with each of the $\infty^{2}$ elements $e$ and personify it by an observer $T$, we can speak of the two-dimensional manifold of elements which $e$ really describes and that which it appears to describe to the observer $T$. It is clearly not necessary, however, that each manifold should contain $\infty^{2}$ distinct elements; it may happen, for instance, that, while the element $e$ assumes $\infty^{2}$ distinct positions in space, the observer $T$ may regard it as assuming only $\infty^{1}$ distinct positions or even as being fixed.

\footnotetext{
* Presented to the Society, August 30, 1929; received by the editors in July, 1928.

† A pair of congruences which are related in this manner are considered by Darboux. (Leçons sur la Thérie Générale des Surfaces, vol. 4, p. 125.)
} 
I propose to prove in this paper that, under certain conditions, a straight line which appears to the observer $T$ to describe a normal congruence does so in reality. I shall then show that certain known theorems on normal congruences can be derived from this result in a simple manner without analysis and that in some cases extensions which I believe to be new suggest themselves.

2. Kinematical preliminaries. When infinitesimal displacements are given to a trihedral $T$ and to a point $P$, there are three displacements associated with the point $P$ :

(i) the actual displacement of the point $P$;

(ii) the displacement which the observer $T$ attributes to the point $P$, or, more briefly, the apparent displacement of $P$;

(iii) the displacement of a point which is fixed with reference to $T$ and which coincides with $P$ when $T$ and $P$ are in their initial positions, or, as we shall call it, the displacement of the moving system at $P$.

We shall recall the familiar fact that the actual displacement of $P$ is the resultant of the apparent displacement of $P$ and the displacement of the moving system at $P$.

Let us now suppose that a two-parameter motion of a trihedral $T$ has been defined by associating a position of $T$ with each pair of values of two parameters $u$ and $v$. Corresponding to each position $(u, v)$ of the trihedral there are certain straight lines which we shall call special lines, and which we may define by saying that a line $l$ is a special line if, in every infinitesima! displacement of $T$ from the position $(u, v)$ to an adjacent position $(u+d u$, $v+d v)$, the displacement of the moving system at each point on $l$ is normal to $l$.

In order to determine these lines we shall make use of the fact that, corresponding to any position $(u, v)$ of $T$, there exists in general a pair of straight lines, which we shall call the axes, such that $T$ may be brought from its initial position $(u, v)$ to any adjacent position $(u+d u, v+d v)$ by a combination of rotations of suitable magnitudes about these two axes.* It is then easy to see that the special lines are the $\infty^{2}$ common transversals of the two axes. The axes may of course be imaginary or coincident, but there are always $\infty^{2}$ real special lines forming a linear congruence.

There is one special case which we shall have to consider: that in which the axes are indeterminate. When this happens every infinitesimal displacement of the trihedral reduces to a rotation about some straight line passing through a certain fixed point and lying in a certain fixed plane through this

* See Mannheim, Principes et Développements de Géométrie Cinêmatique, 1894, p.127, or Darboux, Leģons, vol. 1, p. 91. 
point. The special lines then consist of all lines passing through the point together with all lines lying in the plane. The point and the plane may be both at infinity, and in this case every infinitesimal displacement is a translation parallel to a certain plane, and the special lines that are not wholly at infinity are the lines which are perpendicular to this plane.

Another exceptional case is that in which the trihedral takes only $\infty^{1}$ distinct positions in spite of the fact that we have used two parameters to specify each of its positions. If this happens, there are $\infty^{3}$ special lines corresponding to each position of the trihedral, and they form a linear complex. We shall assume in what follows that this case does not arise.

3. Normal congruences. Considering again the two-parameter motion of a straight line $l$ and the associated trihedral $T$, we can state our fundamental result as follows:

If in each of its positions the line $l$ is a special line with reference to the corresponding position of the trihedral $T$, then $l$ will describe a normal congruence in space during its two-parameter motion if, and only if, the observer $T$ regards it as describing a normal congruence. Further, when $l$ does describe a normal congruence, a point on $l$ which appears to describe a surface cutting all the rays $l$ at right angles does so in reality.

We shall suppose that the straight line $l$ assumes $\infty^{2}$ distinct positions both in reality and as viewed by $T$, and that the congruence which it appears to describe is a normal congruence. We can then find on each ray $l$ a point $P$ such that every apparent infinitesimal displacement of $P$ is normal to $l$. If we take any position of the ray $l$ and consider the corresponding positions of the trihedral $T$ and the point $P$, it is clear that, in any infinitesimal displacement from this position, the apparent displacement of $P$ and the displacement of the moving system at $P$ are both normal to $l$. Hence the actual displacement of $P$, which is their resultant, is also normal to $l$, or, in other words, the locus of the point $P$ in space is a surface which cuts all the rays at right angles.

We may remark that, if we have constructed a normal surface $S$ of the congruence which $l$ actually describes, and if $T$ observes this surface from each of his positions, he will believe that it moves with a two-parameter motion and envelops a surface $S^{\prime}$, and $S^{\prime}$ will be for $T$ a normal surface of the congruence which he believes the ray $l$ to describe. If $T$ were to construct this surface $S^{\prime}$ and to fix it in position in his trihedral, then, from the point of view of a fixed observer, $S^{\prime}$ would move and would envelop $S$.

We have omitted from our proof the case in which one or other of the two congruences under consideration reduces to a system of only $\infty^{1}$ distinct lines or to a single line. If this happens, the other congruence will be a normal 
congruence without further conditions beyond the requirement that each ray should be a special line. We have only to observe that, in the case of a ruled surface or of a single straight line, we can always find a point $P$ on each line such that every displacement of $P$ is normal to the line, remembering that we are entitled to regard a displacement of zero magnitude as being normal to any direction that we please. We shall therefore agree to say that a line describes a normal congruence when it assumes at most $\infty^{1}$ distinct positions.

Our theorem admits the following converse, the proof of which will be evident:

If a ray $l$ describes a normal congruence both in reality and in appearance, and if we can find a point $P$ on the ray in each of its positions so that $P$ both in reality and in appearance describes a surface which cuts all the rays at right angles, then each ray is a special line with reference to the corresponding trihedral.

Our theorem and its converse may be thrown into an alternative form. Let us associate with each position of the moving trihedral a plane element formed by a point of a certain fixed surface and the tangent plane thereat. This element will appear to $T$ to describe a manifold of $\infty^{2}$ plane elements which in general is not formed by the elements of any surface. In order that this manifold should consist of the elements of some surface, it is necessary and sufficient that the line drawn through the point of each plane element perpendicular to its plane should be a special line with reference to the corresponding position of $T$.

4. The theorems of Beltrami and Ribaucour. We shall now point out that, when we take into account a certain elementary property of the motion of a surface which rolls upon an applicable surface, the classical theorems of Beltrami and of Ribaucour on the deformation of congruences may be regarded as almost immediate consequences of our result.*

Consider a pair of applicable surfaces $S$ and $S^{\prime}$ which are not both ruled surfaces, and let $S^{\prime}$ be fixed in space while $S$ rolls upon it so that each point $M$ of $S$ is brought in turn into coincidence with the corresponding point $M^{\prime}$ of $S^{\prime}$, the tangent planes, and corresponding directions in the tangent planes, also coinciding. Let $T$ be any trihedral invariably connected with the rolling surface $S$, and, with each position of $T$, let us associate (a) a ray $l$ passing through the corresponding point of contact of the two surfaces, (b) a ray $m$ lying in the common tangent plane at the point of contact. Now,

* Proofs of these theorems depending on kinematical considerations are given by Darboux (Legons, vol. 3, pp. 348, 352), but they are of a different nature from ours. 
if we consider the congruence which the ray $l$ really describes and subject it to a deformation in Beltrami's sense corresponding to the deformation of $S^{\prime}$ into $S$, we shall clearly obtain a congruence which is identical with that which the ray $l$ appears to describe to the observer $T$. This remark, together with an analogous remark about the ray $m$, shows that, in order to prove the theorems of Beltrami and Ribaucour, we need only assure ourselves that the rays $l$ and $m$ are both special lines with reference to the corresponding position of $T$. This, however, is evident when we remember that every infinitesimal displacement of the rolling surface is a rotation about some line touching both surfaces at their point of contact, so that, in virtue of a remark which we have already made, the special lines consist of all lines passing through the point of contact, together with all lines lying in the common tangent plane at the point of contact.

It will be clear that the proposition given at the close of the preceding section leads in a similar manner to a theorem due to Bianchi* which may be stated as follows: Let us associate a plane element $e$ with each point $M$ of a surface $S$. Let $S$ be deformed and, as it varies, let each of the plane elements move so that it retains an invariable position with reference to the point $M$ and to elements of the surface issuing from $M$. If we suppose that these plane elements were those of some surface $\Sigma$ in their original positions, then, in order that they should still be the plane elements of some surface after deformation, it is necessary and sufficient that each normal to $\Sigma$ should either pass through the corresponding point $M$ of $S$, or else lie in the tangent plane at $M$ to $S$.

We may also remark that Ribaucour's theorem on the deformation of a normal congruence of plane curves lying in the tangent planes of a surface can be derived in like manner from our result.

5. Another theorem of Ribaucour. Our next application will yield a result which, as we shall see, may be regarded as a generalisation of another theorem of Ribaucour.

Consider any two surfaces $S, S^{\prime}$ between whose points $M, M^{\prime}$ a correspondence of any nature has been established, and construct the surface $\Sigma$ which is the locus of the extremity $P$ of a vector $O P$ drawn through a fixed origin $O$ equal and parallel to $M M^{\prime}$. With the points $M$ and $M^{\prime}$ of the two surfaces associate rays $m$ and $m^{\prime}$ respectively, both parallel to the normal to $\Sigma$ at the corresponding point $P$, and so situated that by subjecting them to a common translation we could cause $m$ to pass through $M$ and $m^{\prime}$ to pass through $M^{\prime}$. We shall show that, as $M$ and $M^{\prime}$ describe the surfaces on which

* Rendiconti dei Lincei, (5), vol 24 (1915), p. 4. 
they lie, neither of the two rays can describe a normal congruence unless the other does so also.

We shall hold $S^{\prime}$ fixed in space, and we shall attach a trihedral to $S$ and allow $S$ to move with a two-parameter motion, carrying its associated system of rays invariably fixed to it, so that corresponding points of the two surfaces are in turn brought into coincidence, and so that every position of $S$ may be derived from its original position by a translation. It is easy to see that all infinitesimal displacements from the position in which $M$ and $M^{\prime}$ coincide are translations parallel to the tangent plane to $\Sigma$ at the corresponding point $P$, so that any line parallel to the normal to $\Sigma$ at $P$ is a special line. Now, when $M$ and $M^{\prime}$ coincide, the corresponding rays $m$ and $m^{\prime}$ coincide in a single line which is a special line for the corresponding position of the trihedral. If we identify this line with the line $l$ of our fundamental result, the truth of our assertion will be evident.

We shall now particularise the nature of the correspondence between the two surfaces and we shall suppose that it is a correspondence by parallel tangent planes, so that we can find corresponding nets of curves on the two surfaces such that corresponding curves have the same direction at corresponding points (and in general this can be done in one and only one way). If, further, we note that the tangent planes at the points $M, M^{\prime}, P$ to the respective surfaces are now all parallel, it will be clear that our result may be stated as follows:

If we have traced corresponding nets on two surfaces $S$ and $S^{\prime}$ so that corresponding tangents to curves of the nets are parallel, and if with corresponding points $M, M^{\prime}$ of the two surfaces we associate rays $m, m^{\prime}$ parallel to the common direction of the normals at $M$ and $M^{\prime}$ in such a way that the position of $m$ in relation to the tangents to the net at $M$ is the same as the position of $m^{\prime}$ in relation to the tangents to the net at $M^{\prime}$, then, if one of these rays describes a normal congruence, the other does so also.

The theorem of Ribaucour* to which we have referred need not be enunciated separately, for it is the particular case of what we have just stated which arises when the surfaces have the same spherical representation of their lines of curvature, the nets which we have introduced being identified with the lines of curvature.

6. The moving trihedral of Darboux. The remainder of our work arises out of two theorems which were given by Caronnet, and, in order to establish and to extend these theorems, it will be necessary to make some remarks on the circumstances which arise when we identify the trihedral $T$ of our initial

* Journal de Mathématiques, (4), vol. 7 (1891), p. 40. 
result with the moving trihedral of Darboux. We shall thus associate with each point $M$ of a given surface a trihedral formed by three mutually perpendicular straight lines intersecting in $M$, one of which coincides with the normal to the surface at $M$. The position of the other two edges of the trihedral in the tangent plane may conveniently be specified by tracing on the surface a singly infinite family of curves $\Gamma$ and stipulating that the tangent at a point $M$ to the curve $\Gamma$ that passes through it has an assigned fixed position in relation to the moving trihedral. Since for our purposes two trihedrals are equivalent if one is fixed in relation to the other, we shall not need to know how this assigned fixed position is situated in relation to the trihedral, the fact that it is fixed being all that is relevant. We see, then, that in order to complete the determination of the motion of the trihedral, it is a matter of indifference whether we use a family of curves $\Gamma$ or a family of curves which cut the curves $\Gamma$ at an arbitrary constant angle.

The axes $a_{1}$ and $a_{2}$ of a Darboux trihedral are always real: $a_{1}$ is a line in the normal plane of the line of curvature $C_{1}$ at $M$ passing through the corresponding center of principal curvature $P_{1}$, and $a_{2}$ is a line in the normal plane of the line of curvature $C_{2}$ at $M$ passing through the corresponding center of principal curvature $P_{2}{ }^{*}$ We can easily complete the statement of the position of these axes when the indeterminateness in the motion of the trihedral has been removed by tracing a family of curves $\Gamma$; and to make this clear we shall first show that the center of geodesic curvature of the curve $\Gamma$ which passes through $M$ lies on the straight line which joins the points $A_{1}$ and $A_{2}$ where the axes $a_{1}$ and $a_{2}$ cut the tangent plane.

Draw through $M$ the straight line in the tangent plane which is normal to the curve $\Gamma$ and let it cut the straight line $A_{1} A_{2}$ in $G$. Consider that infinitesimal displacement of the moving trihedral $T$ in which $M$ moves along $\Gamma$. The displacement of the moving system at $G$ is parallel to the normal to the surface at $M$, for it is perpendicular to $A_{1} A_{2}$, which is a special line, and it is also perpendicular to $M G$ because at $M$, and hence at every point on $M G$, the displacement of the moving system is perpendicular to $M G$. Combining this with the displacement along $M G$ which the observer $T$ attributes to the point $G$, we see that the actual displacement of $G$ lies in the normal plane of the curve $\Gamma$ at $M$.

This fact suffices to show that $G$ is the center of geodesic curvature of the curve $\Gamma, \dagger$ for it implies that $G$ lies on the characteristic of the normal plane of $\Gamma$ corresponding to a displacement of $M$ along $\Gamma$, and this characteristic

* Darboux, Leçons, vol. 2, p. 365.

† Cf. Darboux, Lȩ̧ons, vol. 3, p. 117. 
is the polar line of the curve, so that the point $G$ where it meets the tangent plane is the center of geodesic curvature of the curve.

If we trace any system of isogonal trajectories $\Gamma^{\prime}$ of the curves $\Gamma$, it follows similarly that the center of geodesic curvature of the curve $\Gamma^{\prime}$ which passes through $M$ also lies in the line $A_{1} A_{2}$. We may thus construct the line $A_{1} A_{2}$ by joining the centers of geodesic curvature of the curves $\Gamma$ and $\Gamma^{\prime}$ which pass through $M$. The axes of the trihedral are then found by joining the points where this line cuts the normal planes of the lines of curvature to the corresponding centers of principal curvature. In other words, the axes are the polar lines of those isogonal trajectories of the curves $\Gamma$ which touch the lines of curvature at $M$.

Incidentally, we have proved a theorem which is due to Lipka. ${ }^{*}$ We have shown that, if we construct the $\infty^{2}$ isogonal trajectories of a singly infinite family of curves on a surface, the locus of the centers of geodesic curvature of the trajectories passing through a point is a straight line.

7. The theorems of Caronnet. $\dagger$ The first of these states that the necessary and sufficient condition that the line joining the centers of geodesic curvature of the curves of an orthogonal net on a surface should describe a normal congruence is that the corresponding radii of geodesic curvature should be connected by some functional relation.

This theorem was extended by Beal, $\ddagger$ who proved that we may replace the orthogonal net by any net the curves of which cut at a constant angle, and showed that the result remains true in the limiting case in which one of the two centers of geodesic curvature is constantly at infinity.

Caronnet's second theorem states that if, corresponding to each point on a surface, we construct the line joining the center of principal curvature of the line of curvature of the first system at the point to the center of geodesic curvature of the line of curvature of the second system at the point, this line will describe a normal congruence if, and only if, the corresponding radii of principal and geodesic curvature satisfy some functional relation. $\$$

To prove Beal's extension of Caronnet's first theorem, we shall suppose that we are given a net of curves formed by a family of curves $\Gamma$ together with their trajectories $\Gamma^{\prime}$ at some constant angle. The line $l$ joining the centers of geodesic curvature at a point $M$ on the surface of the curves $\Gamma$ and $\Gamma^{\prime}$ is then a special line for the trihedral whose motion has been determined by

* These Transactions, vol. 13 (1912), p. 94.

$\dagger$ Comptes Rendus, vol. 115 (1892), p. 589.

$\ddagger$ American Journal of Mathematics, vol. 35 (1913), p. 24.

$\$$ A proof of this theorem is given by Pell, American Journal of Mathematics, vol. 20 (1898), p. 112. 
means of the curves $\Gamma$ or the curves $\Gamma^{\prime}$ in the manner which we have explained. In order that $l$ should describe a normal congruence, it is therefore necessary and sufficient that the observer $T$ should regard it as doing so. Now, for the observer $T, l$ lies in a fixed plane, and he can therefore regard $l$ as describing a normal congruence only if it assumes at most $\infty^{1}$ distinct positions in relation to his trihedral. The condition that this should be so is clearly the existence of a functional relation between the radii of geodesic curvature of the curves $\Gamma$ and $\Gamma^{\prime}$, for these radii are the intercepts which the line $l$ makes on two lines which are fixed in one of the faces of the trihedral.

We may notice that, if we are given the functional relation, we can at once derive the normal surfaces of the congruence which $l$ describes from the involutes of the curve which $l$ appears to envelop. For instance, we can see immediately the truth of a theorem given by Beal which asserts that the foot of the perpendicular from $M$ on $l$ describes a normal surface of the congruence if, and only if, the functional relation between the radii of geodesic curvature is that which expresses the constancy of their ratio.

In order to discuss Caronnet's second theorem, we shall identify the curves $\Gamma$ which we use to define the motion of the trihedral with one of the families of lines of curvature. The axes $a_{1}$ and $a_{2}$ of the trihedral are now the polar lines of the lines of curvature, ${ }^{*}$ so that $a_{1}$ is the line $P_{1} G_{1}$ in the normal plane of the line of curvature $C_{1}$ which joins the center of geodesic curvature $G_{1}$ of $C_{1}$ to the center of principal curvature $P_{1}$ corresponding to $C_{1}$, and $a_{2}$ is the line $P_{2} G_{2}$ which is similarly related to the line of curvature $C_{2}$.

The theorem in question follows at once, for $P_{1} G_{2}$ is a special line and, as in the preceding theorem, it appears to lie in a fixed plane. The condition that it should describe a normal congruence is therefore the existence of a functional relation between the radii of principal and geodesic curvature $M P_{1}$ and $M G_{2}$.

8. Extensions of Caronnet's second theorem. Caronnet's second theorem leads to the construction of a normal congruence associated with the lines of curvature of a surface only when the surface is of a special type. Our methods, however, suggest the following propositions which are of a similar nature, but which lead to the construction of a normal congruence whatever be the nature of the surface:

The line $G_{1} Q$ joining the center of geodesic curvature of $C_{1}$ at $M$ to a point $Q$ on the polar line of $C_{2}$ describes a normal congruence if $Q$ is so chosen that there exists a functional relation between the length $M Q$ and the radius of geodesic curvature $M G_{1}$.

* Darboux, Leçons, vol. 3, p. 345. 
The line $P_{1} R$ joining the center of principal curvature $P_{1}$ to a point $R$ on the tangent to the corresponding line of curvature $C_{1}$ describes a normal congruence if $R$ is so chosen that there exists a functional relation between the length $M R$ and the radius of principal curvature $M P_{1}$.

The truth of these propositions will be sufficiently evident if we remark that in each case the straight line under consideration is a special line which appears to the observer $T$ to intersect constantly a fixed straight line, and that the only normal congruences in which every ray intersects a fixed straight line are those whose rays are generators of a system of $\infty^{1}$ right circular cones which have this straight line as their common axis.

The second of these two results, however, is equivalent to a theorem of Beltrami,* for, if we look at the matter from the standpoint of the sheet $S_{1}$ of the surface of centers on which $P_{1}$ lies, and remember that the curves which the normals to the original surface touch on $S_{1}$ form a family of geodesics, along whose orthogonal trajectories $M P_{1}$ is constant, we see that our result is equivalent to the statement that, if on any surface $S_{1}$ we trace a family of geodesic parallels and draw through each point of the surface a ray normal to the parallel and cutting the surface at an angle which is constant along each of the parallels, these rays will form a normal congruence.

The following is another result of a similar character:

If a ray $l$ meets the polar lines of the lines of curvature at $R_{1}$ and $R_{2}$ and makes a constant angle with the normal to the surface, the condition that it should describe a normal congruence is the existence of a functional relation between the perpendicular distances of $R_{1}$ and $R_{2}$ from the normal to the surface.

The proof of this proposition will be sufficiently clear if we remark that the only normal congruences in which each ray makes the same angle with a fixed straight line are those whose rays all touch a cylinder having its generators parallel to the fixed straight line.

We may note that this result may be enunciated in a form which involves a reference only to the congruence formed by the normals $P_{1} P_{2}$ and not to a particular normal surface of this congruence. We could, in fact, have equally well described the polar lines of the lines of curvature as the tangent lines to the sheets of the focal surface of the congruence of normals which are conjugate to the ray $P_{1} P_{2}$ of this congruence. $\dagger$ We might also have described them as the rectifying lines of the curves which the rays $P_{1} P_{2}$ touch on the sheets of the focal surface, and, with this alteration in the enunciation, the particular case of our result in which the constant angle

* See Darboux, Leçons, vol. 3, p. 349.

† Darboux, Leçons, vol. 3, p. 335. 
between $l$ and $P_{1} P_{2}$ is a right angle can be extended to congruences which are not normal, but are subject to the condition that the angle between the focal planes is constant. We have in fact the following theorem:

If with each ray $m$ of a congruence in which the angle between the focal planes is constant we associate another ray $l$ which is perpendicular to $m$ and meets the rectifying lines of the curves which the rays $m$ touch on the sheets of the focal surface, the necessary and sufficient condition that the ray $l$ should describe a normal congruence is the existence of a functional relation between the perpendicular distances from $m$ of the points where $l$ cuts the rectifying lines.

Let $P_{1}, P_{2}$ be the foci of the ray $m$, lying respectively on the sheets $S_{1}$, $S_{2}$ of the focal surface, and let the rays touch the curves $\Gamma_{1}$ on $S_{1}$ and the curves $\Gamma_{2}$ on $S_{2}$. With each ray $m$ associate a trihedral $T$ whose motion we shall not specify completely but shall subject only to the condition that the focal planes of the ray $m$ are fixed planes with reference to $T$. The focal planes are the osculating planes of the curves $\Gamma_{1}, \Gamma_{2}$, so that the rectifying planes are the planes through the ray $m$ perpendicular to the focal planes, and are therefore fixed planes with reference to $T$.

We shall show that the special lines which lie in planes perpendicular to $m$ intersect the two rectifying lines. Take any point $M$ on $m$ and through it draw a plane at right angles to $m$. Consider the special line which lies in this plane, and let it cut the rectifying planes of the curves $\Gamma_{1}$ and $\Gamma_{2}$ in $G_{1}$ and $G_{2}$ respectively. If we give to $T$ that infinitesimal displacement in which the focus $P_{1}$ moves along $\Gamma_{1}$ and notice that both the actual and apparent displacements of $M$ lie in that focal plane which osculates $\Gamma_{1}$ at $P_{1}$, we see that the displacement of the moving system at $M$ also lies in this plane and is therefore normal to $M G_{1}$. Arguing as we did in our discussion of the Darboux trihedral, we see that the displacement of the moving system at $G_{1}$ is parallel to the ray $m$, for it is perpendicular to $G_{1} G_{2}$ which is a special line, and it is perpendicular to $M G_{1}$ because at $M$, and hence at every point on $M G_{1}$, the displacement of the moving system is perpendicular to $M G_{1}$. Combining this displacement of the moving system with the displacement in the rectifying plane which the observer $T$ attributes to the point $G_{1}$, we see that the actual displacement of $G_{1}$ also lies in this rectifying plane, so that $G_{1}$ is a point on the rectifying line of the curve $\Gamma_{1}$. Similarly, $G_{2}$ is a point on the rectifying line of the curve $\Gamma_{2}$. Hence those special lines which are perpendicular to the ray $m$ are transversals of the two rectifying lines. It follows then that the line $l$ of our theorem is a special line, and therefore $l$ will describe a normal congruence if, and only if, the observer $T$ regards it as doing so. The truth of our theorem will then be evident if we remember that the condition that a straight line which always remains parallel to a fixed 
plane should describe a normal congruence is that its projection on this plane should have only one degree of freedom.

We may remark in conclusion that, in the course of the foregoing argument, we have incidentally shown that the axes of any trihedral in relation to which the ray $m$ and its focal planes are fixed lie on the paraboloid formed by those transversals of the two rectifying lines which are perpendicular to the ray $m$.

When the congruence formed by the rays $m$ is a normal congruence, this paraboloid becomes identical with the paraboloide des huit droites of Mannheim.*

* Comptes Rendus, vol. 84 (1877), p. 645.

Trintty College, Dublin 